University of New Hampshire

University of New Hampshire Scholars' Repository

Physics Scholarship

Physics

$8-1-2000$

\title{
Association of energetic neutral atom bursts and magnetospheric substorms
}

A. M. Jorgensen

L. Kepko

M. G. Henderson

Harlan E. Spence

Boston University, harlan.spence@unh.edu

G. D. Reeves

See next page for additional authors

Follow this and additional works at: https://scholars.unh.edu/physics_facpub

Part of the Physics Commons

\section{Recommended Citation}

Jorgensen, A. M., L. Kepko, M. G. Henderson, H. E. Spence, G. D. Reeves, J. B. Sigwarth, and L. A. Frank (2000), Association of energetic neutral atom bursts and magnetospheric substorms, J. Geophys. Res., 105(A8), 18753-18763, doi:10.1029/1999JA000445.

This Article is brought to you for free and open access by the Physics at University of New Hampshire Scholars' Repository. It has been accepted for inclusion in Physics Scholarship by an authorized administrator of University of New Hampshire Scholars' Repository. For more information, please contact Scholarly.Communication@unh.edu. 


\section{Authors}

A. M. Jorgensen, L. Kepko, M. G. Henderson, Harlan E. Spence, G. D. Reeves, J. B. Sigwarth, and L. A. Frank 


\title{
Association of energetic neutral atom bursts and magnetospheric substorms
}

\author{
A. M. Jorgensen, ${ }^{1}$ L. Kepko, ${ }^{2}$ M. G. Henderson, ${ }^{1}$ H. E. Spence, ${ }^{3}$ \\ G. D. Reeves, ${ }^{1}$ J. B. Sigwarth, ${ }^{4}$ and L. A. Frank ${ }^{4}$
}

\begin{abstract}
.
In this paper we present evidence that short-lived bursts of energetic neutral atoms (ENAs) observed with the Comprehensive Energetic Particle and Pitch Angle Distribution/Imaging Proton Spectrometer (CEPPAD/IPS) instrument on the Polar spacecraft are signatures of substorms. The IPS was designed primarily to measure ions in situ, with energies between 17.5 and $1500 \mathrm{keV}$. However, it has also proven to be a very capable ENA imager in the range $17.5 \mathrm{keV}$ to a couple hundred $\mathrm{keV}$. It was expected that some ENA signatures of the storm time ring current would be observed. Interestingly, IPS also routinely measures weaker, shorter-lived, and more spatially confined bursts of ENAs with duration from a few tens of minutes to a few hours and appearing once or twice a day. One of these bursts was quickly associated with magnetospheric and auroral substorm activity and has been reported in the literature [Henderson et al., 1997]. In this paper we characterize ENA bursts observed from Polar and establish statistically their association with classic substorm signatures (global auroral onsets, electron and ion injections, $A L$ drops, and $\mathrm{Pi} 2$ onsets). We conclude that $\sim 90 \%$ of the observed ENA bursts are associated with classic substorms and thus represent a new type of substorm signature.
\end{abstract}

\section{Introduction}

Energetic neutral atoms (ENAs) are generated when an energetic ion interacts with a cold, neutral, geocoronal particle in a charge exchange reaction. During charge exchange an electron is transfered from the cold geocoronal particle to the hot ion, generating an energetic neutral atom and a cold ion. The charge exchange process preserves the momentum of the ion, and therefore the energetic neutral will contain information about the pitch angle and energy of the original ion. The ENA will then travel in a straight line from the point where it was generated. This later property allows the ENA source region to be imaged through their remote detection.

\footnotetext{
${ }^{1}$ Los Alamos National Laboratory, Los Alamos, New Mexico.

${ }^{2}$ Department of Earth and Space Sciences, University of California, Los Angeles.

${ }^{3}$ Center for Space Physics, Boston University, Boston, Massachusetts.

${ }^{4}$ Department of Physics and Astronomy, University of Iowa, Iowa City.
}

Copyright 2000 by the American Geophysical Union.

Paper number 1999JA000445.

0148-0227/00/1999JA000445\$09.00
The earliest associations of ENAs with ring current activity was published by Moritz [1972], who suggested that short-lived populations of energetic particles at

low altitude were created through charge exchange with oxygen of ENAs created in the ring current region. Mizera and Blake [1973] were able to corroborate this by extending measurements down to $12.4 \mathrm{keV}$, well below the lower-energy threshold of $250 \mathrm{keV}$ of Moritz [1972], and showing that the spectrum of the low-altitude population was consistent with a ring current source through charge exchange.

Direct measurements of ENAs were first reported by Hovestadt and Scholer [1976]. They suggested that highly anisotropic energetic hydrogen measurements were observations of ENAs generated by charge exchange of ring current particles with the tenuous hydrogen geocorona. The first comparison of direct ENA measurements with ring current activity was made by Roelof et al. [1985]. They analyzed ENA measurements from IMP $7 / 8$ and ISEE 1 and demonstrated, using hourly averages, a rough correspondence between ENA count rate and the rate of ring current recovery as measured by the $D$ st index. Jorgensen et al. [1997] observed a similar proportionality between the ENA escape rate from the ring current and the $D s t$ index using ENA data from the Polar satellite.

The first ENA image of a storm time ring current was published by Roelof [1987]. More recently, Lui 
et al. [1996] have reported on the first direct composition measurements of ENAs. They reported data from a single Geotail pass around the dayside of the Earth, in which the ring current ENA flux was observed continuously for $\sim 12$ hours. Results from the lowaltitude Swedish ASTRID/PIPPI ENA imager have also been reported [Barabash et al., 1997; Brandt et al., 1997, 1999].

The first ENA images from Polar were reported by Henderson et al. [1997]. The images revealed the unprecedented statistical and temporal coverage of the CEPPAD/IPS instrument [Blake et al., 1995] and showed it to be an extremely capable ENA imager. That paper presented several cases of ENA measurements of the storm time ring current and examples of preliminary analysis of the images. In addition to the expected magnetic storm signatures, the paper reported a single case of a short-lived ENA signature that was well correlated with classic magnetospheric signatures of substorms.

Indeed, substorm phenomenology includes the sudden appearance of energetic particles in the near-Earth tail region at onset [e.g., DeForest and Mcllwain, 1971]. The hot ion component then drifts westward while dispersing. On the basis of this known ion phenomenology during substorms, the expected ENA signature would consist of an initial brightening in the near Earth tail region near onset, then a rapid earthward [Reeves et al., 1996] or tailward [Ohtani et al., 1992] expansion of this region. This would be followed by a slow westward drift of the hot injected ions and thus of a similarly evolving patch of ENA emission. Finally, as the ions drift farther west and disperse, the region of ENA emission should expand and diminish in brightness. The substorm ENA response should be much shorter-lived than the storm ENA response owing to the much shorter timescales. Most importantly, though, is that the enhancement of hot ions in the inner magnetosphere is an established part of substorms, and therefore we should expect that substorms will generate a burst of ENAs. What was not known prior to the launch of Polar, however, was how intense these substorm ENAs were, and whether it would be possible to measure them with any current or planned instrumentation.

In this paper we will adopt the nomenclature of ENA "burst" for any Polar ENA signal that is too short-lived to be associated with build up and decay of the ring current. A burst is defined to be any ENA signal that lasts $<6$ hours. In practice, observed bursts tend to last of the order 2 hours or less. First, we establish the intrinsic statistical properties of the ENA bursts; then we correlate them with classic substorm signatures. We conclude that $\sim 90 \%$ of the ENA bursts are associated with classic substorms.

\section{Data Set}

The IPS instrument was designed to measure energetic ions in the range of $15-1500 \mathrm{keV}$. It has nine look directions, looking outward from the spacecraft in a plane containing the spin axis, and separated from each other by $20^{\circ}$. Each look direction can sample up to 32 times (sectors) per spin, yielding up to 288 "pixels" sampled in a single 6-s spin, covering the complete sky, as shown in Plate 1a. In this paper we will use data from the instrument's integral channel and study the period October 8, 1996, to October 7, 1997. During this interval the integral channel energy range was $17.5-1500 \mathrm{keV}$ and sampled at 32 sectors per spin in the seven central look directions and at 16 sectors per spin in the two near-spin-aligned look directions. This data set was integrated over 16 spins (96 s) and consisted of the seven central look directions sampled at 32 sectors, so the data set could be visualized as an array in three dimensions $(7 \times 32 \times N)$, where $\mathrm{N}$ is the number of 16 spin blocks included. To improve statistics, this data set was smoothed in time with a boxcar window of five 16-spin blocks ( $8 \mathrm{~min})$. The instrument is not capable of directly distinguishing between ions and ENAs. The identification of ENAs is inferred when there is a highly anisotropic component arriving from the direction to the Earth and a very low isotropic background. Thus a key reason for the high quality of the Polar ENA data is the orbit of Polar. It is highly eliptic with a $9 R_{E}$ apogee and a $1.8 R_{E}$ perigee. The apogee is located in the northern polar cap. In this region (and in the southern polar cap) the flux of energetic ions is very low, allowing global ENA images to be recorded on a regular orbital basis. This ability to regularly construct global ENA images allows for global imaging studies that were heretofore impossible.

It was expected that the IPS would measure some neutrals from the storm time decay of the ring current. This had also been done in the past with similarly built instruments [Hovestadt and Scholer, 1976; Lui et al., 1996; Roelof et al., 1985; Roelof, 1987]. However, it turned out that the IPS instrument is so sensitive to low particle fluxes that it was capable of observing ENAs from charge exchange of the Earth's ring current, not only when it was enhanced from storm activity but also during geomagnetically quiet periods. Wilken et al. [1997] did not observe a statistically significant ENA signal from the quiet time ring current with the High Energy Particle - Low energy particle Detector (HEPLD) instrument on Geotail, but were able to compute an upper limit that was in reasonable agreement with their expectations.

In addition to the expected ENA signature of geomagnetic storms [Henderson et al., 1997, 1999; Jorgensen et al., 1997], shorter-lived bursts of ENAs were observed, which were too short-lived to be storm related and which were much more localized than the global storm signature. The IPS records these emissions after as little as $2-8-\mathrm{min}$ integrations. This period is sufficiently short to be able to observe the onset and evolution of these ENA burst structures. One such event, that occurred on July 31,1996 , was shown to be related to a magnetospheric substorm [Henderson et al., 1997]. 
Another event that took place on November 24, 1996, was reported by Henderson et al. [in press, 1999].

In this paper we study particularly the onset characteristics of the short-lived ENA bursts. It is very important to note that we selected the ENA burst intervals only using the IPS ENA data. Any subsequent comparison of these data, described next, with other coincident substorm indicators is thus accomplished without any selection bias.

\section{Analysis}

Because of the difficulty of writing an automatic procedure for identifying ENA bursts, we browsed our data and manually selected ENA bursts. To facilitate this analysis, a special summary plot format was created to enhance the relatively low count rate ENAs (see Plate $1 b$ ). The summary plots have a logarithmic color scale ranging from 0.5 to 10 counts/s per pixel, the range of count rates most typically observed in these ENA bursts. As with storm time ENAs, the Polar IPS can unambiguously measure ENA bursts only when the ion foreground is inherently low. These intervals occur primarily at times close to apogee and for a brief time near perigee when Polar traverses the high-latitude lobe regions. Nevertheless, when in these regions, there exists a weak background due both to cosmic rays and weak ion fluxes that is removed before plotting. In most cases, both of these sources can be treated as isotropic. In contrast, the ENA fluxes come predominantly from regions close to the Earth and are thus inherently anisotropic. The background non-ENA count rate was taken to be the median count rate over all pixels at a given time. This isotropic background was then subtracted from the data before plotting it in the summary plot format (Plate $1 \mathrm{~b}$ ).

The format of the summary plot is as follows. As mentioned before, the two spin-aligned look directions are omitted from this analysis because when Polar is in a favorable location for observing ENAs, they rarely look in the right directions for observing them. The remaining seven look directions were plotted in the summary plot in separate panels. To the left of each panel is written the angle between the spacecraft positive spin axis and each look direction. Each look direction samples 32 sectors in one spin, and in each panel the sectors are displayed vertically from sector 0 at the bottom of each panel to sector 31 at the top of each panel. The gray areas identify those regions where the ion foreground was considered too large to yield reasonable counting statistics on the ENA signal. This happens twice per orbit when Polar passes through the radiation belts and additionally when occasional energetic ions are observed in the polar cap. The maximum allowed background ion count rate was set to 10 counts/s. While this value was selected somewhat arbitrarily, the results are not affected much by it, as the background count rate is usually either much higher than 10 counts/s, or much lower than 10 counts/s. In other words, there are few borderline cases where the background is similar to the ENA count rate.

A feature that can be seen easily in the summary plot is the Sun response. The IPS sensors are photosensitive and are "blinded" momentarily after viewing the Sun or the illuminated Earth. The Sun response is usually confined to sector 0 and 1 (and occasionally sector 31 as well), because the sectoring system is defined in such a way as to put sector 0 pointing toward the Sun. Depending on the orientation of the spacecraft (which in turn depends on season), the Sun response will appear in one or more of the look directions. In Plate $1 \mathrm{~b}$ the sun response can be seen in the $150^{\circ}$ and $130^{\circ}$ look directions. Another feature that can be seen is the Earth response, which is a line of bright pixels slowly crossing sectors with time in the $90^{\circ}$ look direction. Depending on distance to the Earth and season (during Northern Hemisphere summer the northern polar region is more bright for example), the Earth response may also appear in adjacent look directions. In Plate $1 \mathrm{~b}$ the Earth shine also appears in parts of the $70^{\circ}$ and $110^{\circ}$ look directions. The predictable earth light and sunlight features are well-known instrumental artifacts. They provide a good visual reference since ENAs are primarily observed to come from locations near the Earth, and when processing for ENAs they can be ignored.

The ENA bursts were identified visually by looking for regions that were at the same time enhanced above background by at least $0.5-1$ counts/s (though more often 1-3 counts/s) and were directionally localized, which means that they appeared as approximately horizontal stripes several pixels wide across the summary plots. Such an example can be seen in Plate 1b. An ENA burst starts at $1135 \mathrm{UT}$ and ends at 1345, while another ENA burst starts at 1440 and ends after 1600 UT. The uncertainty on the start times determined from the summary plots were of the order of $15 \mathrm{~min}$.

In order to narrow down the uncertainty on the onset time and get an idea of the spatial location of the ENA bursts, we produced ENA images around the time of onset, as determined from the summary plots, at 96-s resolution, and smoothed in time with a 5-point box car $(8 \mathrm{~min})$. Examples of ENA images surrounding the ENA burst at 1130 UT on March 7, 1997, are shown in Plate 1c. In this format the pixelized ENA data during each measurement interval are projected onto the Solar Magnetic (SM) $X-Y$ plane. When Polar is at sufficiently high altitude above the magnetic equator these projections will give some indication of the source region of the measured ENAs. Naturally, care must be taken, as ENA images in reality are a line-of-sight integral of the directional volume emission rate of a translucent object. However, if care is taken, a great deal of information can be derived from this type of projection.

By inspecting these projection plots it was then possible to more precisely identify a start and end time to within the 96-s resolution of the data set. Note that 


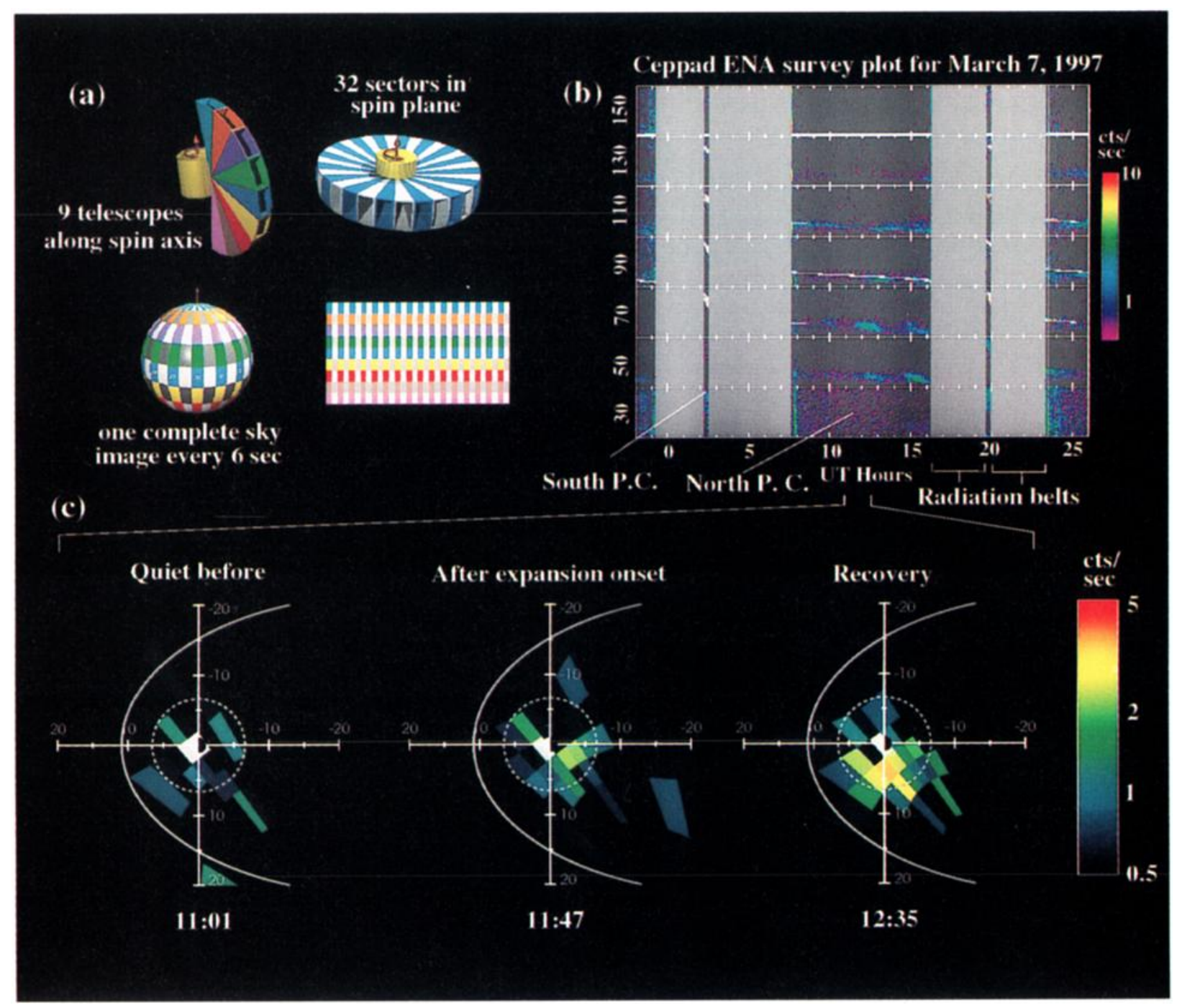

Plate 1. (a) The IPS instrument has nine look directions, arranged perpendicular to the spin axis. As the satellite spins, each look direction samples up to 32 times (sectors) per 6-s spin for a total of up to 288 pixels covering the complete sky. (b) An example of the summary plot format that was used for identifying energetic neutral atom (ENA) bursts. The 7 panels contain data for the 7 central look directions. In each panel the 32 sectors are plotted from bottom to top. The light gray line stretching across the $90^{\circ}$ look direction (and parts of the 100 and $70^{\circ}$ ) is Earth-shine. The ENA signal is concentrated around this Earth-shine line since ENAs are emitted from near the Earth. We can see one ENA burst starting at $1130 \mathrm{UT}$ and ending at 1345 UT and a second burst starting at $1440 \mathrm{UT}$, and ending after $1600 \mathrm{UT}$. (c) Three ENA images near the 1130 UT burst, one before onset, one shortly after onset, and one during the recovery phase. Onset occurred in the premidnight region at $1130 \mathrm{UT}$ (center image), and the bright ENA patch then drifts with the prevailing ion drift speed around dusk toward noon (rightmost image) 


\section{VIS Earth Camera}

07 Mar $1997(97 / 066)$

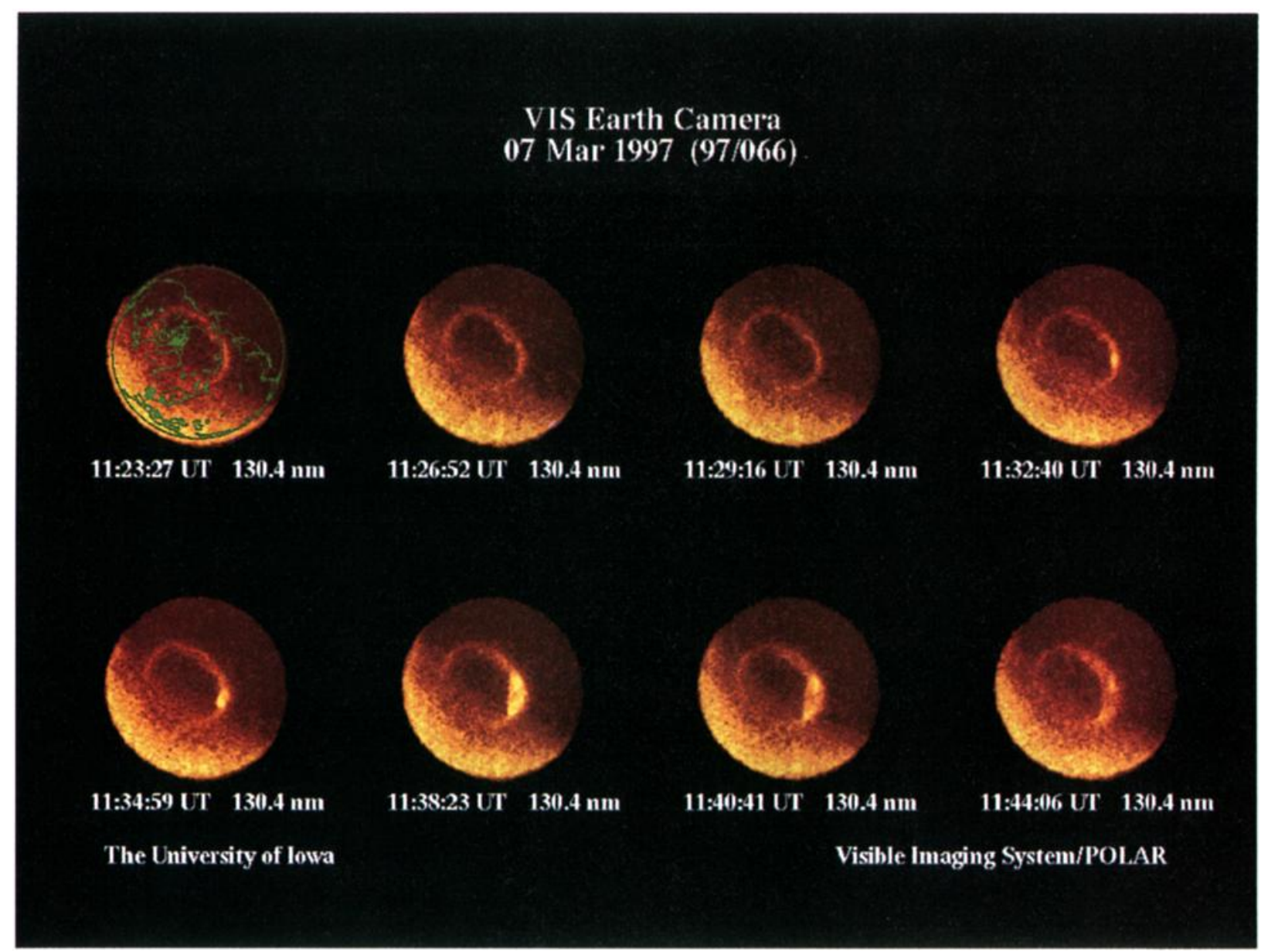

Plate 2. A series of VIS images around the time of the ENA burst onset at 1130 UT on March 7,1997 . The auroral onset occurred at $\sim 1133 \mathrm{UT}$. 


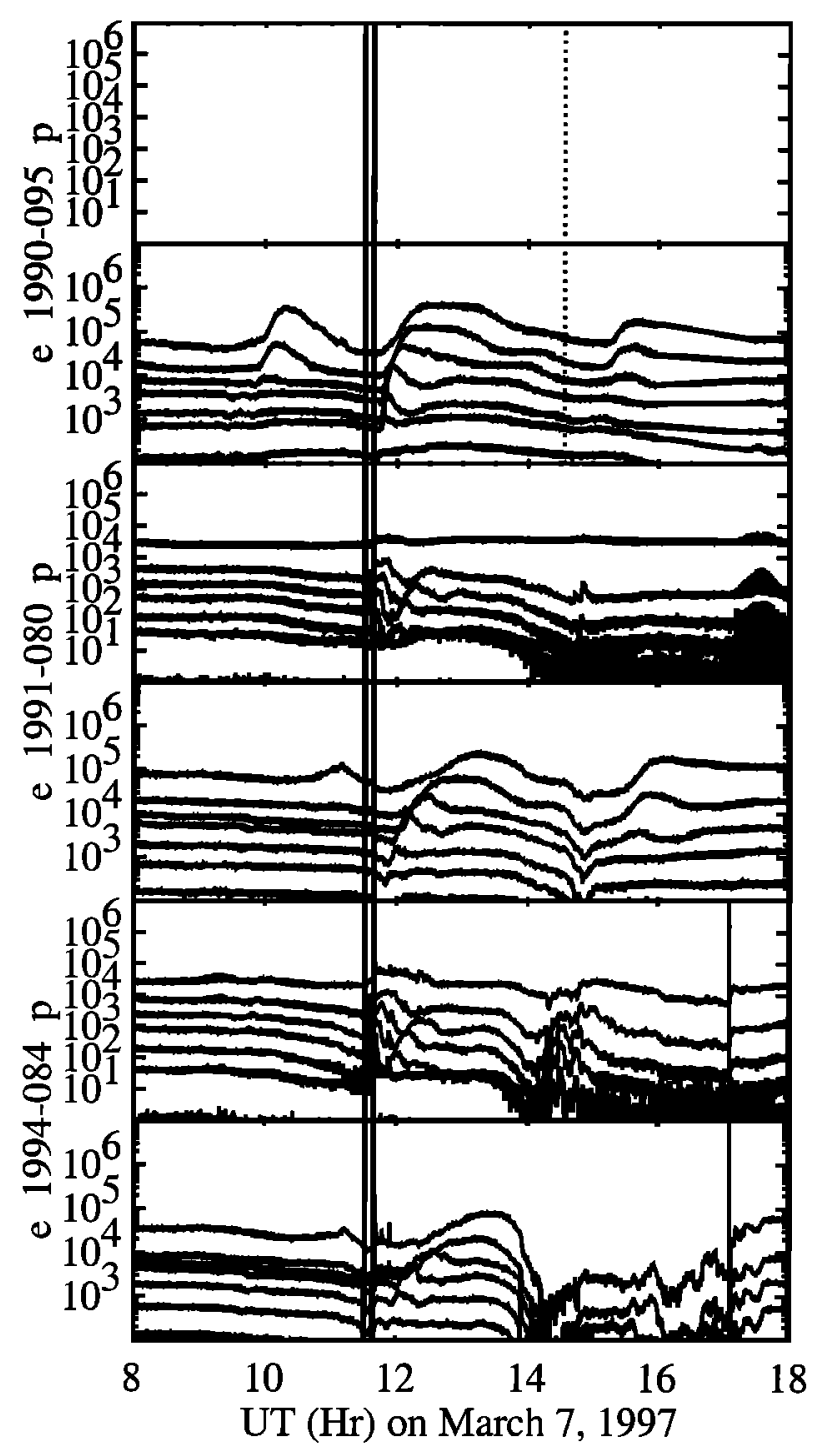

Figure 1. The Los Alamos National Laboratory (LANL) energetic particle data around the time of the ENA burst onset at 1130 UT on March 7, 1997. The ion injection occurs at $1131 \mathrm{UT}$ and the electron injection occurs at $1139 \mathrm{UT}$, as marked by two vertical solid lines. The points where the satellites pass the noon meridian are marked by a vertical dotted line. The points where they pass the midnight meridian is marked by a vertical solid line.

because IPS can only observe ENAs from part of its orbit, it was not always possible to identify the start/onset and end time of the ENA bursts. If Polar entered a high ion population before an ENA burst ended, we could not ascribe an accurate end time. We excluded events for which we were not able to ascribe a clear onset time. In addition to identifying the onset time, we also identified the onset region, which we defined as the R-MLT box in the SM $X-Y$ plane that contained the pixel or collection of pixels that first brightened. For example, for the ENA burst shown in Plate 1, we found that the onset occurred at 1129:35 UT, and inside a region bounded by 5 and $9 R_{E}$, and 1900 and 2100 MLT.
For each ENA burst identified we next searched for classic substorm signatures. We visually surveyed data from one of the auroral imagers on the Polar satellites, the Visible Imaging System (VIS) [Frank et al., 1995]. We used the 5-min average images from the Earth camera to identify auroral substorm expansion onsets. Plate 2 shows a series of VIS images around the time of the ENA burst onset in Plate $1 b$. In this case the auroral onset was determined to occur near $1133 \mathrm{UT}$. We also looked through the Los Alamos National Laboratory geosynchronous energetic particle data (LANL) [Higbie et al., 1978; Baker et al., 1985; Belian et al., 1992] for both electron and ion injection onset, plotted in Figure 1 for the time around the same ENA burst, and noted whether they were dispersionless or not. In this case a dispersionless ion injection occurred at $1131 \mathrm{UT}$, and a dispersionless electron injection occurred at 1139 UT. Additionally, we searched for two classic ground-based substorm signatures. We surveyed the provisional $A L$ index [Davis and Sugiura, 1966] produced by WDC-C2 Kyoto (see Figure 2), and searched it for substorm signatures. We defined a substorm signature to be a sharp negative turning of $A L$. We measured the time at which the negative turning occurred (with a typical accuracy of $15 \mathrm{~min}$ ), as well as the size of the drop in nanoteslas. Finally, we identified Pi2 onsets surrounding the measured ENA onsets using the three components of the Kakioka magnetic field data. We looked for polarized power within $\pm 30 \mathrm{~min}$ of the ENA onset, and defined a $\mathrm{Pi} 2$ onset as having polarized power 2 standard deviations above the average for that interval, with a minimum amplitude of $0.2 \mathrm{nT}$. In Figure 3 we show the raw and Pi2 bandpass filtered data from the Kakioka magnetometer. Onset time was calculated using the method described above.

\section{Results and Discussion}

During the 1-year study period October 8,1996 , to October 7, 1997, we found a total of 141 clear ENA burst onsets. Since the orbit of Polar is not synchro-

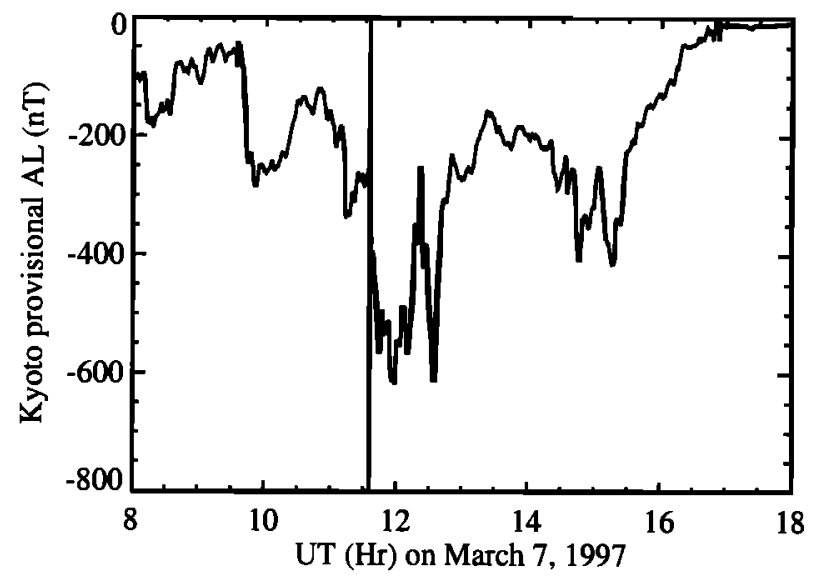

Figure 2. The provisional $A L$ index produced by WDC-C2 Kyoto. A sudden drop in $A L$ occurred at 1135 UT. 


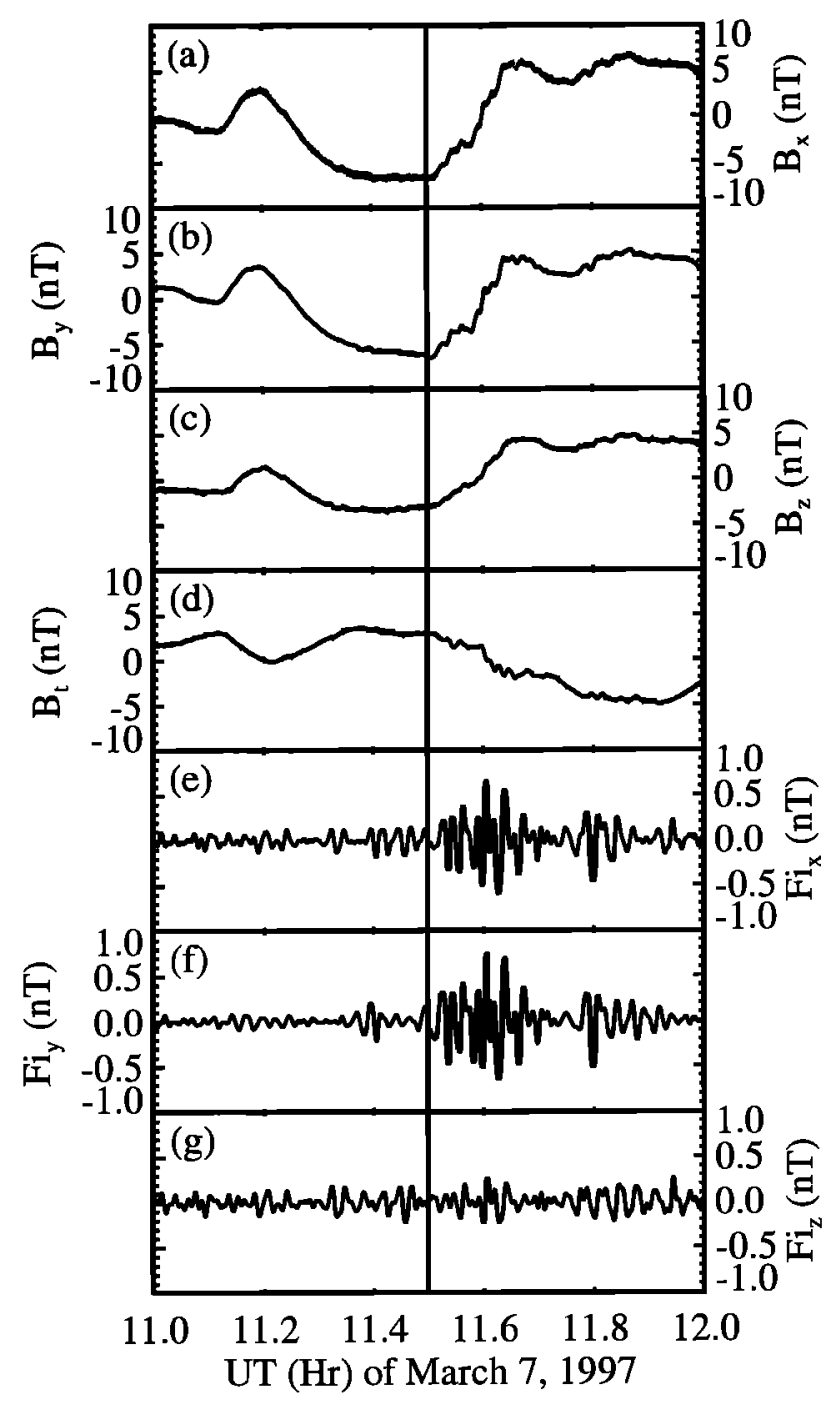

Figure 3. The Kakioka Pi2 data for the March 7, 1997, 1130 UT ENA burst: (a)-(c) $x, y, z$ components of the magnetic field; (d) variation of the total magnetic field; (e)-(f) magnetic field filtered to the Pi2 bandpass (40$150 \mathrm{~s})$. The Pi2 onset can be seen to occur at 1130 UT.

nized with the Earth's rotation, there should be no UT bias of identified ENA bursts, and we do in fact find that the measured ENA bursts are distributed approximately uniformly in UT with 6 ENA bursts in each UT hour interval. The monthly distribution showed more variation, with $\sim 13$ bursts/month for October through January, 24 bursts/month in February and March, and $\sim 6$ bursts/month in April through September. The genesis of this distribution is not clear. During the same period the possible observing time remained nearly constant, although it was slightly reduced in the early parts of 1997 . The possible observing time was computed as the number of 16-spin (96-s) blocks for which the computed background was smaller than 10 counts/s, and for which the ring current was not enhanced due to a storm. We attribute the reduction during early 1997 partly to several storms that occurred during that period. However, the reduction during this period is in the wrong sense, and too small, to account for the variation in ENA burst frequency. ENA observations are possible approximately half the time. Thus the 141 clear ENA burst onsets observed implies that $\sim 300$ clear ENA bursts, as selected by our criteria, occurred during the 1-year study period.

The local time distribution of the ENA bursts is shown in Figure 4. This plot was generated in the following way: If an ENA burst was found to accur in $X$ hourly local time sectors, the histogram that counts occurrence would have each of those sectors incremented by $1 / X$. In this way the sum over all histogram bins is equal to the total number of ENA bursts. The solid line in Figure 4 represents the distribution of ENA bursts. The dotted line represents the distribution of dispersionless ion injections as measured by Lopez et al. [1990] (see their Figure 4). The two distributions are very similar. This is what we expect if the ENA bursts are a remotely sensed signature of the injection.

We attempted a similar comparison of the projected radial distribution of ENA bursts with the radial distribution of injections from the Lopez et al. [1990] study. We did not find a good correspondence. The projected position of the ENA signal was significantly closer to the Earth. There are two reasons for this. The first is that the density of the geocorona drops off rapidly with distance from the Earth, so that for a given inward moving injection front, it will not be observed until it reaches further in to the inner magnetosphere. This would cause the ENA bursts to be observed closer to the Earth. Another reason is that an isotropic ion distribution on a given field line will tend to emit more ENAs from near the foot of the field line because those extend deeper into the geocorona. When these off-equatorial portions

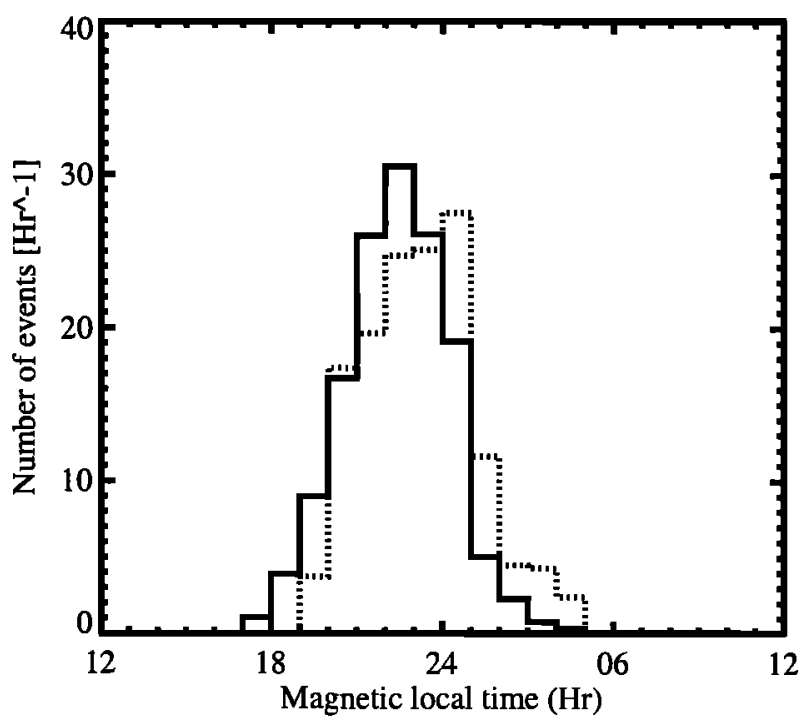

Figure 4. The solid line represents the MLT distribution of measured ENA bursts. The dotted line represents the distribution of dispersionless ion injections as measured by Lopez et al. [1990] (see their Figure 4). 


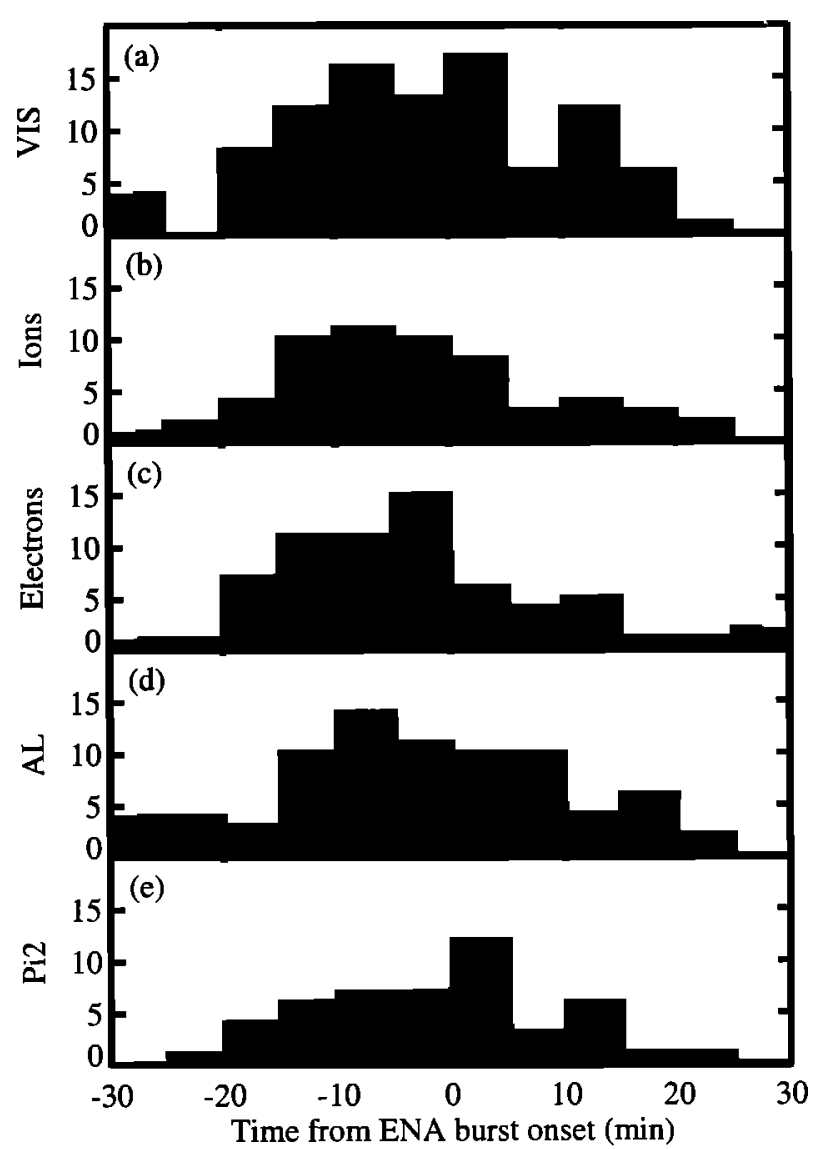

Figure 5. Distribution of time from ENA burst onset to (a) VIS onset, (b) dispersionless ion injection, (c) dispersionless electron injection, (d) negative $A L$ turning, and (e) Pi2 burst onset. VIS onsets occurred $\sim 2$ min earlier, ion injections $\sim 4-5$ min earlier, electron injections $\sim 5$ min earlier, AL onsets $\sim 4$ min earlier, and $\mathrm{Pi} 2$ bursts $\sim 1-2 \mathrm{~min}$ earlier. Typically, $\sim 90 \%$ of the occurred within $\pm 20 \mathrm{~min}$ of the ENA burst onsets.

of the field line are projected onto the equatorial plane (as seen near Polar apogee) they appear closer to the Earth, but this is purely a projection effect.

We also found good correspondence between ENA bursts and the other classic substorm signatures explored in this paper. Figure 5 summarizes time differences between the ENA burst onset time and the other classic substorm onset signatures described previously: visible auroral onset, geostationary injection of ions and electrons, sharp $A L$ drops, and $\mathrm{Pi} 2$ intensifications. These differences in timing are described below.

The average time difference from an ENA burst to a signature is

$$
<\Delta t>=\frac{\sum_{\imath=1}^{N} t_{s}^{i}-t_{E N A}^{i}}{N}
$$

with an uncertainty of

$$
\sigma_{<\Delta t>}^{2}=\frac{\left(\sigma_{s}\right)^{2}}{N}+\frac{\left(\sigma_{E N A}\right)^{2}}{N}
$$

where $t_{s}^{i}$, and $t_{E N A}^{i}$ are the classic signature, and ENA onset times respectively for the $i$ th event, with uncertainties $\sigma_{s}$, and $\sigma_{E N A} . N$ is the number of events. In the following we will quote the average (and median when different) time differences, with uncertainty on the average time difference following in parenthesis.

The VIS distribution, for which 114 events had VIS data available, is shown in Panel a. The vast majority, 99 of the 114 or $87 \%$, were associated with an auroral substorm onset as measured by the VIS instrument on Polar. VIS onsets occurred $\sim 2(0.3)$ min earlier than ENA burst onsets, and $91 \%$ of them occurred within \pm 20 min of the ENA burst onset.

The distribution of LANL ion injections is shown in Panel b, and that of electron injections in Panel $\mathrm{c}$. LANL ion data were available for 134 events. Of these events, $89(66 \%)$ had ion injections, while $120(89 \%)$ had electron injections. The ion injections preceded the ENA burst by 4-5(0.2) min, while the electron injections came $\sim 5(0.16)$ min before the ENA burst. Again, $88 \%$ and $89 \%$ of the ion and electron injections, respectively, occurred within 20 min of the ENA burst.

The quick look AL data set that we used commences in January 1997, and thus we have limited coverage of AL. Consequently, 102 ENA bursts had $A L$ data available, and of those $81(79 \%)$ were associated with a sudden negative turning of $A L$. The negative $A L$ turnings occurred $\sim 4(1.1)$ min earlier than the ENA burst, and $92 \%$ of them occurred within $\pm 20 \mathrm{~min}$.

Kakioka station is best suited for $\mathrm{Pi} 2$ detection between 1000 and $2000 \mathrm{UT}$, when the station is near midnight. Only 67 events fulfilled this criterion. Of those, $50(75 \%)$ had an associated Pi2 onset. The Pi2 onsets occurred 1-2(0.2) min before the ENA burst, and $92 \%$ of them occurred within \pm 20 min.

It is interesting that all the classical substorm signatures occur on average 1 to $5 \mathrm{~min}$ before the onset of the ENA burst. The travel time for a $20 \mathrm{keV}$ ENA from the Earth to the Polar satellite $8 R_{E}$ away is $\sim 30 \mathrm{~s}$. The

Table 1. Summary of the association Between the 141 Clear ENA Burst Onsets, and Classic Substorm Onset Signatures.

\begin{tabular}{lrrr}
\hline & Data & Event & Fraction \\
\hline VIS onsets & 114 & 99 & $87 \%$ \\
LANL p-inj & 134 & 89 & $66 \%$ \\
LANL e-inj & 134 & 103 & $77 \%$ \\
LANL p or e & 135 & 120 & $88 \%$ \\
AL drop & 102 & 81 & $79 \%$ \\
Pi2 & 67 & 50 & $75 \%$ \\
All but Pi2 & 80 & 57 & $71 \%$ \\
All & 37 & 21 & $57 \%$ \\
p 18-24 & 65 & 51 & $78 \%$ \\
p 20-22 & 30 & 26 & $87 \%$ \\
e 00-06 & 59 & 56 & $95 \%$ \\
e 02-04 & 20 & 20 & $100 \%$ \\
\hline
\end{tabular}

Here $\mathrm{p} / \mathrm{e} \mathrm{XX}-\mathrm{YY}$ signifies ion/electron injection when at least one satellite was between XX and YY MLT. 
bounce time for the corresponding ion can be from 45 sec to 2 min typically (5-20 keV parallel energy, 6.6$10 R_{E}$ L-shell). The bounce time may well be a typical timescale from the onset of equatorial substorm activity to the arrival of the injected ions in the denser atmosphere closer to the Earth. When combined these two time scales can account for nearly all of the delay from $\mathrm{Pi} 2$ onsets, and a smaller fraction of the delays from the other classic substorm signatures.

While coverage is fairly easy to judge for the VIS images (VIS typically sees the whole auroral oval, or has a data gap), it is much harder to estimate accurately for the other data types. For the LANL injections we initially assumed coverage if any of the satellites were taking data at the time, regardless of their local time. However, this is not a sufficient criterion for detection of an injection if one occurred [Lopez et al., 1990]. If we restrict data coverage to times when a satellite was in the pre-midnight region for ions, or post-midnight regions for electron, detection of injections is greatly improved. For example, if we require a satellite to be located between 2000 and 2200 MLT, the fraction of ENA bursts that have ion injections increases from 66 to $87 \%$. If we require a satellite to be located between 0200 and 0400 MLT, all ENA bursts measured had an electron injection. These results are summarized in Table 1. A similar situation is found for $\mathrm{Pi} 2$ onsets, where we from the outset restricted attention to 1000-2000 UT when Kakioka station was on the nightside. If we consider all times, then only $60 \%$ of ENA bursts are associated with a Pi2 onset. Figure 6 shows the UT distribution of ENA bursts that did (solid) and did not (dotted)

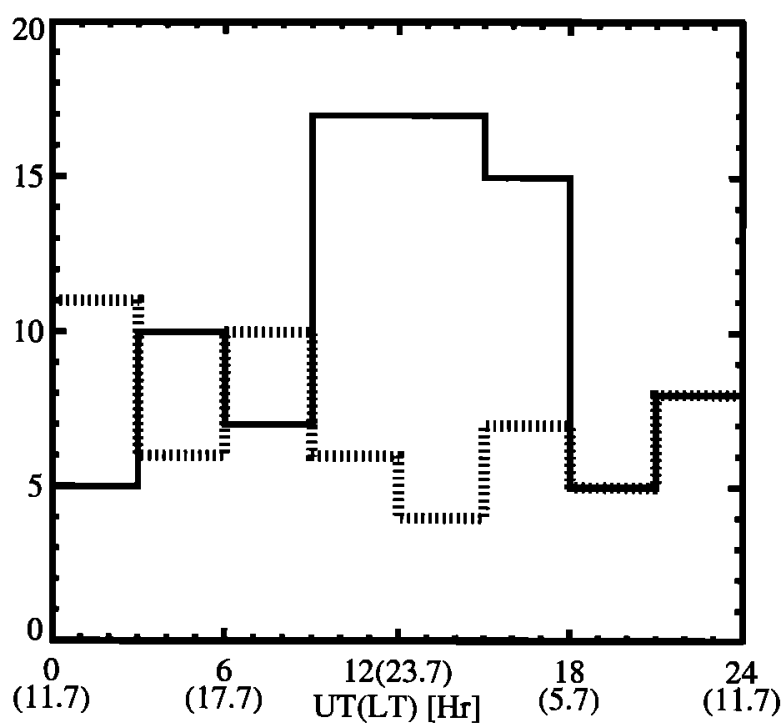

Figure 6. UT distribution of Kakioka station for positive (solid line) and negative (dotted line) associations with ENA bursts. Notice that the when Kakioka observed $\mathrm{Pi} 2$ in association with ENA bursts, it was more likely to be located near midnight, whereas when it did not observe $\mathrm{Pi} 2$ it was more likely to be located away from midnight.
Table 2. Distribution of UT of ENA Bursts for Which an $A L$ Signature Was Observed.

\begin{tabular}{cccc}
\hline Region & Data & Events & Percent \\
\hline $0000-0400$ UT & 20 & 18 & 90 \\
$0400-0800$ UT & 18 & 13 & 72 \\
$0800-1200$ UT & 20 & 19 & 95 \\
$1200-1600$ UT & 16 & 11 & 69 \\
$1600-2000$ UT & 15 & 13 & 87 \\
$2000-2400$ UT & 13 & 7 & 54 \\
\end{tabular}

It can be seen that the chance of detecting an $A L$ onset is larger between 0000 and 1200 UT than between 1200 and 2400 UT.

have an associated Pi2 onset. Table 2 lists the fraction of ENA bursts associated with $A L$ onsets for different UT intervals. It can be seen that the probability of detecting an $A L$ onset is larger before 1200 UT than it is after $1200 \mathrm{UT}$. The reason for this is that the $A E$ stations are more densely spaced in the western hemisphere (between $0^{\circ}$ and $180^{\circ} \mathrm{W}$ longitude, see Figure 7 ). These stations will then be clustered around midnight near 700 UT.

For a given ENA burst and a given classic substorm signature the chance of observing the classic signature in association with the ENA burst is $\sim 90 \%$. Of the 80 ENA bursts for which data were available for all classic substorm signatures, only $2(2.5 \%)$ did not show any signature. On the other hand, $70(88 \%)$ had at least

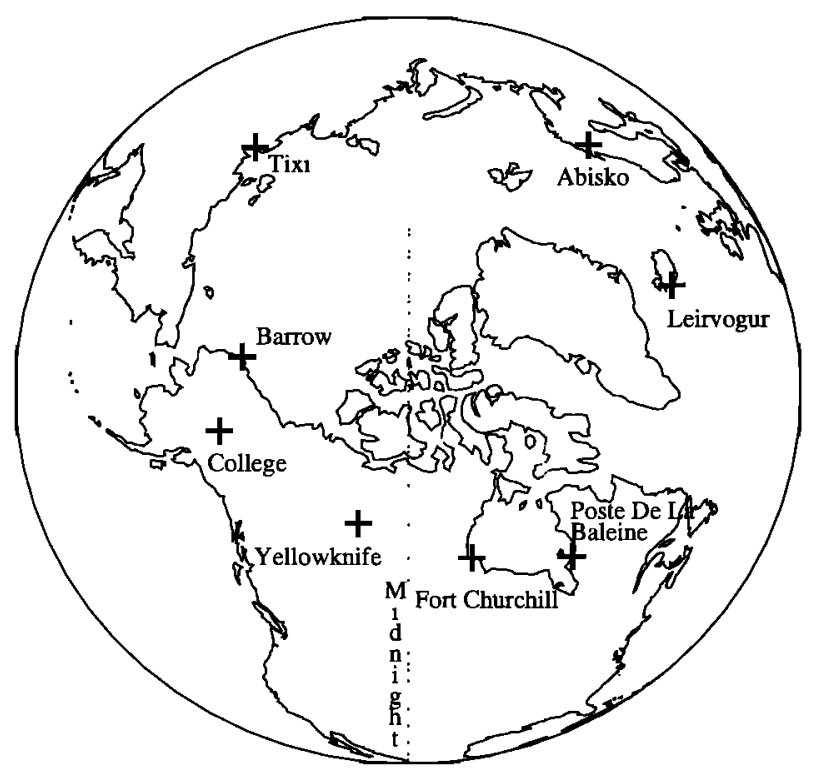

Figure 7. Longitude and latitude distributions of ground magnetometer stations that contribute to the version of the $A E$ index used in this paper. The plot is a globe projection, and the dotted line shows the midnight meridian for $0700 \mathrm{UT}$. It can be seen that more stations are located in the premidnight region around $0700 \mathrm{UT}$, than at other times 
2 signatures, and $60(75 \%)$ had at least three signatures. The average number of signatures observed for each ENA burst was 3.3. The remaining $\sim 10 \%$ can be ascribed to insufficient coverage and/or imperfect identification of the signatures. Another possibility is that they are in fact not substorms, but some other process that cause enhancement of the ion population in the midnight inner magnetosphere (and thus ENA bursts). Auroral onsets are often considered one of the most reliable substorm signatures (and in fact the original definition of a substorm). In this case we observe VIS onsets for $\sim 90 \%$ of the ENA bursts. The 15 ENA bursts that did not show VIS onsets do not appear to have any morphological differences from those for which a VIS onset was observed. We also find that 7 out of $8(88 \%)$ of those events had no Pi2 onset, further indicating that these were not substorms. Four of $7(57 \%)$ had no electron injection, and 2 of $7(29 \%)$ had no ion injection. The lack of electron and Pi2 signatures do indicate that these are ENA enhancements that have a lesser correspondence with classic substorm signatures. However, the fraction that does see ion injections (71\%) is only slightly lower than that for the overall population (78\%), indicating that these ENA bursts are indeed associated with ion injection-like signatures in the midnight region. We conclude that $\sim 90 \%$ of the observed ENA bursts are associated with classic substorms, while $10 \%$ are associated with other processes that cause enhancements of the $\geq 20 \mathrm{keV}$ ion population in the midnight region. Some possibilities include ULF wave activity and short-term enhancements in the convection electric field not associated with substorms.

\section{Conclusion}

We found 141 clear ENA bursts in the year October 8, 1996, to October 7, 1997. The MLT distribution of the onset of these ENA bursts agrees very well with previous findings [Lopez et al., 1990] of the location of substorm associated dispersionless energetic ion injections. On a case-by-case basis we find that nearly $90 \%$ of the ENA bursts are associated with one or more of the classic substorm signatures: auroral onset, geosynchronous energetic particle injection, auroral electrojet enhancement, $\mathrm{Pi} 2$ pulsation onsets. An average of 3.3 of these classic substorm signatures are observed in association with an ENA burst, and $75 \%$ of ENA bursts were associated with at least three signatures. We conclude that $~ 90 \%$ of the observed ENA bursts are signatures of substorms, while the remaining $10 \%$ are signatures of some other process that causes a sudden enhancement in the $\geq 20 \mathrm{keV}$ ion population around midnight.

Acknowledgments. Work at Los Alamos National Laboratory was conducted partially under the auspice of the U. S. Department of Energy, with support from the NASA ISTP and SR\&T programs. Work at UCLA was supported by the National Science Foundation under grant ATM 9314239 and the Los Alamos Division of the Institute of Geo- physics and Planetary Physics, UCRP 708. Work performed at Boston University was supported by NASA grants NAG57706 and NAG5-8039. This research was supported in part at the University of Iowa by the National Aeronautics and Space Administration under NAG5-7712. Kakioka magnetic field data were provided by the Kakioka magnetic observatory. The quick look AE data was provided by the World Data Center C2, Kyoto. The authors wish to thank the referees for several useful suggestions.

Janet G. Luhmann thanks Stas Barabash and Joseph F. Fennell for their assistance in evaluating this paper.

\section{References}

Baker, D. N., W. Aiello, J. R. Asbridge, R. D. Belian, P. R. Higbie, R. W. Klebesadel, J. G. Laros, and E. R. Tech, Los Alamos energetic particle sensor system at geostationary orbit, 85-0243, 1985.

Barabash, S., P. C. Brandt, O. Norberg, R. Lundin, E. C. Roelof, C. J. Chase, B. H. Mauk, and H. Koskinen, Energetic neutral atom imaging by the ASTRID microsatellite, Adv. Space Res., 20(4-5), 1055-1060, 1997.

Belian, R. D., G. R. Gisler, T. Cayton, and R. Christensen, High- $Z$ energetic particles at geosynchronous orbit during the great solar proton event series of October 1989, $J$. Geophys. Res., 97, 16,897-16,906, 1992.

Blake, J. B., et al., Ceppad. Comprehensive Energetic Particle and Pitch Angle Distribution Experiment on Polar, Space Scr. Rev., 71, 531-562, 1995.

Brandt, P. C., S. Barabash, O. Norberg, R. Lundin, E. C. Roelof, C. J. Chase, B. H. Mauk, and M. Thomsen, ENA imaging from the Swedish micro satellite ASTRID during the magnetic storm of 8 February, 1995, Adv. Space Res., 20(4-5), 1061-1066, 1997.

Brandt, P. C., S. Barabash, O. Norberg, and R. Lundin, Energetic neutral atom imaging at low altitudes from the Swedish microsatellite Astrid: Images and spectral analysis, J. Geophys. Res., 104, 2367-2379, 1999.

Davis, T. N., and M. Sugiura, Auroral electrojet activity index $A E$ and its universal time variations, J. Geophys. Res., 71, 785-801, 1966.

DeForest, S. E., and C. E. McIlwain, Plasma clouds in the magnetosphere, J. Geophys. Res., 76, 3587-3611, 1971.

Frank, L. A., J. B. Sigwarth, J. D. Craven, J. P. Cravens, J. S. Dolan, M. R. Dvorsky, P. K. Hardebeck, J. D. Harvey, and D. W. Muller, The visible imaging-system (VIS) for the polar spacecraft, Space Sci. Rev., $\gamma_{1}, 297-328$, 1995.

Henderson, M. G., G. D. Reeves, H. E. Spence, R. B. Sheldon, A. M. Jorgensen, J. B. Blake, and J. F. Fennell, First energetic neutral atom images from Polar, Geophys. Res. Lett., 24, 1167-1170, 1997.

Henderson, M. G., G. D. Reeves, K. R. Moore, H. E. Spence, A. M. Jorgensen, J. F. Fennell, J. B. Blake, and E. C. Roelof, Energetic neutral atom imaging with the Polar CEPPAD/IPS instrument: Initial forward modeling results, Physics and Chemistry of the Earth Part $C$ - Solar-Terrestrial and Planetary Science, 24(1-3), 203$208,1999$.

Henderson, M. G., et al., Polar CEPPAD/IPS energetic neutral atom (ENA) images of a substorm injection, $A d v$. Space. Res, in press, 1999.

Higbie, P. R., R. D. Belian, and D. N. Baker, High-resolution energetic particle measurements at $6.6 R_{E}: 1$ electron micropulsations, J. Geophys. Res., 88, 4851-4855, 1978.

Hovestadt, D., and M. Scholer, Radiation belt-produced energetic hydrogen in interplanetary space, J. Geophys. Res., 81, 5039-5042, 1976.

Jorgensen, A. M., H. E. Spence, M. G. Henderson, G. D. 
Reeves, M. Sugiura, and T. Kamei, Global energetic neutral atom (ENA) measurements and their association with the Dst index, Geophys. Res. Lett., 24, 3173-3176, 1997.

Lopez, R. E., D. G. Sibeck, R. W. McEntire, and S. M. Krimigis, The energetic ion substorm injection boundary, J. Geophys. Res., 95, 109-117, 1990.

Lui, A. T. Y., D. J. Williams, E. C. Roelof, R. W. McEntire, and D. G. Mitchell, First composition measurements of energetic neutral atoms, Geophys. Res. Lett., 23, 2641$2644,1996$.

Mizera, P. F., and J. B. Blake, Observations of ring current protons and low altitudes, J. Geophys. Res., 78, 1058$1062,1973$.

Moritz, J., Energetic protons and low equatorial altitudes, Z. Geophys., 38, 701-717, 1972.

Ohtani, S., S. Kokubun, and C. T. Russell, Radial expansion of the tail current disruption during substorms: A new approach to the substorm onset region, J. Geophys. Res., 97, 3129-3136, 1992.

Reeves, G. D., R. W. H. Friedel, M. G. Henderson, A. Korth, P. S. McLachlan, and R. D. Belian, Radial propagations of substorm injections, in Proceedings of ICS-3, Versailles, France, 12-17 May 1996, ESA SP-339, pp. 579-584, ESA publications division, Keplerlaan 1, Noordwijk, Netherlands, 1996.

Roelof, E. C., Energetic neutral atom imaging of a storm- time ring current, Geophys. Res. Lett., 14, 652-655, 1987. Roelof, E. C., D. G. Mitchell, and D. J. Williams, Energetic neutral atoms (E $\sim 50 \mathrm{keV})$ from the ring current: IMP 7/8 and ISEE 1, J. Geophys. Res., 90, 991-1008, 1985.

Wilken, B., I. A. Daglis, A. Milillo, S. Orsini, T. Doke, S. Livi, and S. Ullaland, Energetic neutral atoms in the outer magnetosphere: An upper flux limit obtained with the hep-ld spectrometer on board geotail, Geophys. Res. Lett., 24, 111-114, 1997.

A. M. Jorgensen and G. D. Reeves, Space and Atmospheric Sciences (NIS-1), MS D466, Los Alamos National Laboratory, Los Alamos, NM 87545

L. Kepko, Department of Earth and Space Sciences, University of California, Los Angeles, CA 90095

M. G. Henderson, Space and Remote Sensing Sciences (NIS-2), MS D436, Los Alamos National Laboratory, Los Alamos, NM 87545

H. E. Spence, Center for Space Physics, Boston University, 725 Commonwealth Ave, Boston, MA 02215

J. B. Sigwarth and L. A. Frank, Department of Physics and Astronomy, University of Iowa, Iowa City, IA 52242

(Received December 6, 1999; revised January 27, 2000; accepted January 31, 2000.) 